|| Print ISSN: 2589-7837 || Online ISSN: 2581-3935 ||

International Journal of Medical Science and Diagnosis Research (IJMSDR)

Available Online at www.ijmsdr.com

NLM (National Library of Medicine ID: 101738824)

Review Article

Volume 5, Issue 10; October:2021; Page No. 34-41

\title{
Minimal Invasive Dentistry: An Emerging Trend
}

\section{Poonam Sharma ${ }^{1}$ Preeti Dhawan ${ }^{2}$ Sumeet Rajpal ${ }^{3}$ Nikhil Bhagat ${ }^{4}$}

${ }^{1}$ P.G Student, Department of Paedodontics and Preventive Dentistry, B.R.S Dental College and Hospital Panchkula, Haryana, India.

${ }^{2}$ Professor, Department of Pedodontics and Preventive Dentistry, B.R.S Dental College and Hospital

Panchkula, Haryana, India.

${ }^{3}$ Professor and Head, Department of Pedodontics and Preventive Dentistry, B.R.S Dental College and Hospital, Panchkula, Haryana, India.

${ }^{4}$ P.G Student, Department of Conservative Dentistry and Endodontics, B.R.S Dental College and Hospital, Panchkula, Haryana, India.

\section{Conflicts of Interest: Nil}

Corresponding author: Dr. Poonam Sharma

DOI: https://doi.org/10.32553/ijmsdr.v5i10.862

\begin{abstract}
:
When first introduced, the term Minimal Intervention Dentistry (MID) referred to use of smaller and more conservative cavity preparations. Today, concept is much broader, being a philosophy of care related to risk assessment of individual patients, early detection of oral disease, targeted preventively orientated strategies and limited surgical intervention appropriate to level of disease $\mathrm{e}^{1-3}$. "Extension for prevention" which was previously taught has been changed to "Prevention of extension".

Though concept of MID has been stressed upon for more than a decade, but its uptake has not been global. MID should be the standard of care in modern dentistry, as it avoids over-zealous restorative interventions as well as supervised neglect. As dental profession worldwide grapples with challenges of dealing with high rates of dental diseases in socially disadvantaged and medically compromised patients, it is even more important to incorporate these concepts into practice to deal with challenge of management of oral diseases. This review focuses on the concept and techniques of minimal invasive dentistry as an emerging trend in paediatric dentistry.
\end{abstract}

Keywords: paediatric dentistry, MID

\section{Introduction:}

With an approach to" nib the evil in bud", Minimal Intervention Dentistry (MID) is modern medical approach to management of caries, utilizing caries risk assessment, focusing on early prevention and interception. Moving focus away from restoration of teeth allows maximum intervention with minimal invasive treatments ${ }^{1}$. This conservative philosophy is more important in pediatric patients as it not only reduces restorative procedure time but also pain and stress, eventually decreasing patient anxiety.

\section{Evolution of the Concept of Minimal Invasive Dentistry}

The advent of fluorides greatly contributed to development of the MID philosophy. Fluoride not to act pre-eruptively, as was thought, but mostly post-eruptively by changing mineral saturation 
characteristics at tooth surface, hence led to a change in cariology paradigm ${ }^{2}$

Development of various adhesive materials systems further contributed to primary aim of MID. The ability to reduce need for cutting away healthy tooth tissues when using adhesive materials, relative to traditional restorative concepts, led to smaller and less destructive cavity preparations and therefore, smaller restorations. ${ }^{3}$

The 'Caries Balance' is determined by relative weight of sums of pathological factors and protective factors. A structured caries risk assessment carried out helps target risk factors, understand behaviour of dental plaque micro flora so that disease activity is reduced, and lesion remineralization can occur ${ }^{4}$.

\section{The Rationale}

It is now widely recognized that dental caries is fundamentally an imbalance between hard tissues and oral microbial salivary environment. The application of minimally invasive dentistry can be justified on grounds that no restorative material can adequately replace natural tooth structure for the long- term and hence its preservation is of paramount importance. ${ }^{5}$

Contributing factors to dental caries particularly in infants and very young children which affect pathogenicity of plaque biofilm include frequency of oral hygiene, pattern of eating, in terms of both fermentable carbohydrates and acid challenges, level of fluoride exposure etc. ${ }^{6}$ Recurrent caries is most common reason for failure of restorations in children, so it becomes imperative to move clinical focus away from art and science of restoring and re -restoring teeth ravaged by dental caries, to prevention of disease and its early interception.

Moreover, conventional dentistry focussed solely on fixing immediate problem is and failed to serve long-term health concerns which often eclipses consequences that contribute to chronic illness. ${ }^{55}$

Recognizing that restorations are not final solution for caries, there is a need for a newer dental care concept based on assessment of caries risk; application of current therapies to prevent, control, and treat the disease with a biologic, therapeutic, or medical model. A major benefit of this approach for both patient and practitioner is that inevitable failures because of unending cycles of recurrent caries is prevented ${ }^{7}$.

\section{Principles of MID}

The four core principles of Minimal Invasive Dentistry are as follows: -

- Recognition: Early identification and assessment of potential caries risk factors

- Reduction: Eliminate or minimize caries risk factors by altering lifestyle and oral environment

- Regeneration: Arrest and reverse incipient lesions, using appropriate measures

- Repair: Conservative caries removal to repair and retain tooth structure.

\section{Recognition}

Caries diagnosis can be considered as a three-step procedure: detection of lesion, followed by an assessment of severity, followed by an assessment of activity of lesion. In children, long before cavitation occurs, caries disease starts because of exposure to risk factors. The purpose of early examination is to detect visually, change in colour, translucency, and structure of enamel. An initial inspection, tooth by tooth, on wet surfaces can spot cavities and brown or white stains. Periodontal status and restorations may also be checked initially. ${ }^{8}$

\section{Methods:}

a) Visual tactile detection

b) Radiographic methods

c) Use of caries assessment indices

d) Use of plaque disclosing dyes

e) Advanced diagnostic aids

f) Caries risk assessment

g) Classification of caries

\section{Reduction}

Once the diagnosis is established optimal preventive measures are undertaken to reduce or eliminate detrimental risk factors by altering diet and lifestyle habits, thereby increasing $\mathrm{pH}$ of oral environment. 
This is accomplished by following MID strategies:

1. Use of Preventive/ prophylactic plaque control agents

2. Oral health education

3. Preventive resin restoration OR pit and fissure sealant application

Preventive/ prophylactic plaque control agents: chemical agents with antimicrobial activity, used to control plaque and provide a considerably preventive action against caries and gingivitis. ${ }^{9}$ Enzymes such as dextranase, mutanase, proteases etc have potential to disrupt plaque matrix and dislodge bacteria from tooth surface while glucose oxidase \& amyl glucosidase enhance host defence mechanism by interfering with metabolism of $S$. mutans. Chlorhexidine, Benzalkonium chloride and, more particularly, Cetylpyridinium chloride are by far most studied and effective antiseptic for plaque inhibition and the prevention of gingivitis. Cetylpyridinium chloride is used in a wide variety of antiseptic mouth rinse products particularly effective against plaque and gingivitis in special health care needs children. Phenols,essential oils, Bisbiquanide antiseptics, Quaternary ammonium compounds ,detergents, acidified sodium chloride etc have been used in mouth- rinses and lozenges. ${ }^{10}$

\section{Oral health education and dietary modifications}

The relationship between carious lesion development and consumption of fermentable sugars strongly supports need for a sound oral health education in children and their guardians as carious process is strongly associated with dietary practices. A high-frequency sugar consumption, prolonged or night-time bottle-feeding practices, sleeping with a bottle etc all are important risk factors, that eventually cause early childhood caries $^{11,12}$. Nutrition education and counselling can be summed up as:

- Reducing high-frequency exposures to sugars; restricting sugar-containing snacks that are slowly eaten.
- Discouraging the behaviour of a child sleeping with a bottle

- Limiting cariogenic foods to mealtimes while promoting snacking with noncariogenic foods

- Rapidly clearing cariogenic foods from child 's oral cavity by tooth brushing or by consumption of protective foods

Evidence suggests that the use of sugar-free chewing gum like xylitol, sorbitol immediately after meals reduces carious lesion progression. Xylitol-based candies and lozenges can be implemented, with good compliance from both children and parents, in a caries- control regimen at schools ${ }^{9}$.

Along with nutritional factors, a comprehensive approach to preventing dental caries in children must include good oral hygiene, appropriate use of fluorides, access to preventive and restorative dental care. High sugar containing foods generally are low in essential nutrients and may be substituted for more nutritious foods in a child ' $\mathrm{s}$ diet. General nutrition recommendations uniformly suggest that sugar should be a small component of the diet. ${ }^{13}$

Anticipatory Guidance is a pro-active and tailormade approach for educating parents in an organized way for a more successful implementation of preventive dentistry. It usually begins with prenatal counselling and proceeds in an age defined manner thus corroborating with different stages of tooth development ${ }^{11}$.

\section{Preventive resin restoration $O R$ pit and fissure sealant application}

Application of preventive resin restorations or fissure sealants is a conservative approach towards confined, incipient occlusal caries in young permanent teeth whereby restoration occurs with a minimum tooth preparation. It aims to seals caries, halt microleakage, and eventual risk of tooth destruction ${ }^{5}$ Loss of restoration and subsequent replacement proves to be less invasive. However, decision to use a sealant must be based on the personal risk and in particular, risk of tooth surface towards carious involvement. 


\section{Regeneration}

During initial stages disease progression can be arrested or reversed using appropriate regenerative agents.

Fluoride can be providedsystemically, or topically by professionals and self-application devices. The fluorides increase caries resistance may arise from both systemic and topical applications byincreased enamel resistance, increased rate of enamel maturation, remineralization of incipient caries, interference with micro- organisms and improved tooth morphology. ${ }^{21}$ The action of fluoride in remineralization is hence considered as the 'gold standard'.

In recent times three calcium phosphate-based remineralization systems have been developed and are now commercially available.Toothpastes based on unstabilized amorphous calcium phosphate (ACP), deliver calcium and a phosphate salt via a dual-chamber device. As salts mix with saliva, they release ions. The mixing of ions results in immediate precipitation of ACP or, in presence of fluoride ions, amorphous calcium fluoride phosphate (ACFP). These phases (ACP and ACFP) are potentially very unstable and rapidly transform into a more thermodynamically stable, crystalline phase such as hydroxyapatite and fluorhydroxyapatite ${ }^{14}$.

Casein phosphopeptide (CPP) is a milk-derived phosphoprotein that stabilizes high concentrations of calcium and phosphate ions, forming nanoclusters of CPP-stabilized ACP or ACFP nanocomplexes. Once in subsurface lesion, these nanocomplexes release weakly bound calcium and phosphate ions, which get deposited into crystal voids. In presence of fluoride, mineral formed in enamel lesion is consistent with fluorapatite or fluorhydroxyapatite. CPP-ACP nanocomplexes also bind into supragingival plaque to increase level of bioavailable ions and function as remineralization and caries prevention agent by creating supersaturation of ions in oral biofilm, modifying dynamics of demineralizationremineralization events when cariogenic challenge occurs. In addition, enzymic breakdown of CPP produces a plaque $\mathrm{pH}$ rise through production of ammonia. ${ }^{15}$.

Application of tricalcium phosphate (TCP) in toothpaste and other remineralizing systems such as varnishes and mouthrinses combines fluoride and functionalized TCP. Functionalized TCP is a tailored, low-dose calcium phosphate system,incorporated into single-phase aqueous or non-aqueous topical fluoride formulation(dentifrice, gel, rinse or varnish). Functionalization serves two major roles: it provides a barrier that prevents premature TCPfluoride interactions, and provides targeted delivery of TCP when applied to teeth. Thus, results in fluoride-based nucleation 'seeding' activity, with subsequent remineralization driven by dietary salivary calcium and phosphate ${ }^{16}$.

NovaMin ${ }^{\circledR}$ (Calcium Sodium Phosphosilicate Bioactive Glass) toothpaste treats hypersensitivity by physical occlusion of exposed dentinal tubules. When introduced into oral environment, it releases sodium, calcium, and phosphate which interact with oral fluids and form crystalline hydroxycarbonate apatite layer,structurally and chemically similar to natural tooth mineral. The ions are protected by glass, trapped for localized $\operatorname{action}^{16,17}$.

Nanohydroxyapatite Toothpaste is a bioactive and biocompatible toothpaste with potential to remineralize by directly filling up micropores. It acts as a template in remineralization by continuously attracting calcium and phosphate ions to enamel, thus promoting crystal integrity and growth. ${ }^{16}$

Arginine bicarbonate is an amino acid complex with particles of calcium carbonate, commercially available for caries control and hypersensitivity. When calcium carbonate dissolves slowly, released calcium is available to remineralize while release of carbonate may give a slight local $\mathrm{pH}$ rise.

\section{Ozone Therapy}

The use of Ozone in dentistry is not only considered to be caries preventive but additionally to be a noninvasive approach to treat. The therapy 
includes differential diagnosis, risk assessment \& documentation; microbial killing, remineralization; final sealing defect, with a micro filling.Ozone treatment alleviates concerns of fear $\&$ apprehension with use of handpiece and local anesthesia and change perrception of dental treatment as a whole ${ }^{18}$.

\section{Repair}

Conservative caries removal \& bioactive restorative approach.

When cavitation is present and surgical intervention is required, conservative caries removal is carried out to maximize repair potential of tooth and retain tooth structure. Bioactive materials are used to restore the tooth and promote internal healing of dentine and prevent iatrogenic pulpal injury. Minimal Intervention Dentistry aims to limit unnecessary removal of healthy tooth structure, and hence, repair of defective restorations is one of its strategies ${ }^{19}$.

\section{Minimal Invasive Interventions}

\section{(a)Atraumatic Restorative Treatment (ART)}

It is a procedure based on removing carious tooth tissues using hand instruments alone and restoring cavity with an adhesive restorative material ${ }^{20}$.

\section{(b)Halls Technique}

It is a method for biological sealing of carious lesions in primary molars. Hall technique is considered a promising restorative option with high acceptability and longevity as caries will be inactive after bacteria are sealed. It also increases child 's compliance and operator comfort as local anaesthesia is eliminated ${ }^{21}$.

\section{CConservative Cavity preparation}

Recently, more conservative forms of operative intervention have been recommended that concentrate on removal of carious dentin and preservation of as much sound tooth structure as possible. The tunnel technique aims to remove and restore proximal dentinal caries via an occlusal access and has theoretical potential to preserve overlying proximal marginal ridge. Proximal box- only or slot preparations include no occlusal dovetail or extension for prevention. ${ }^{22}$

\section{(d)Micro-preparation techniques}

It involves several cutting modalities like micropreparation and fissurotomy burs; Sonic tooth preparation; Air abrasion; Chemo mechanical methods; and Lasers.

Micro-preparation and fissurotomy burs with magnification, allow very precise preparation of teeth. Sonic tooth preparation utilizes the vibrational energy of ultrasonically vibrated metal tips, rather than rotation. It allows precise minimal cutting preparation using diamond coated tips.

\section{(e) Chemico mechanical caries removal}

a non-invasive alternative for removal of carious dentine by applying a solution onto decayed dentinal tissue, allowing it to soften, and finally, scraping it off with blunt hand instruments. Caridex involves intermittent application of pre heated N-monochloro-DLaminobutyric acid to disrupt collagen in carious dentin, thus facilitating its removal. The mechanism of softening involved chlorination of remaining partially degraded dentinal collagen.Carisolv TM, Papacarie or Papain acts by breaking partially degraded collagen molecules, contributing to degradation and elimination of fibrin mantle formed by carious process $^{23}$.

\section{(f)Air abrasion}

The role of air abrasion in micro dentistry is not to amputate sound tooth structure but to preserve as much as possible of the structural integrity remaining around a lesion. A stream of aluminium oxide particles generated from compressed air $(40$ to $160 \mathrm{psi}$ ) or bottled carbon dioxide or nitrogen gas is used for striking abrasive particles (27 or 50 $\mu \mathrm{m}$ in diameter )with high velocity to remove small amounts of tooth structure ${ }^{24}$.

\section{(g)Lasers}

Laser in Paediatric dentistry is an alternative instrument that either complements or substitutes traditional techniques. The idea of substituting drill with a laser instrument which works on hard tissue with no contact, no vibration, no noise and 
less pain has brought about introduction of this device in Paediatric Dentistry. The use of lasers for. Sub ablative CO2 laser irradiation of young healthy teeth could be an effective method for caries prevention and removal of oral mucosal lesions has specific application in Paediatric Dentistry. The carious detection device has proved useful as an additional method for occlusal caries detection. The bactericidal effect of Er: YAG laser irradiation could boost interest in the already widely accepted pits and fissures sealing procedures with therapeutic possibility of treating early childhood caries of enamel with $\mathrm{Nd}$ : YAG laser $^{25}$. The laser though, slow method of caries excavation but with a low level of over preparation supports minimally invasive concepts. In paediatric endodontics, lasers are indicated for pulp capping; pulpotomy and root canal disinfection. Laser-assisted therapy can offer new treatment possibilities in dental trauma in children. LLLT (low level laser therapy) has an important pain-reducing and bio stimulating effect with acceleration of reparative processes that has considerable clinical importance, especially in patients with compromised immune system ${ }^{26}$.

\section{(h)Adhesive Restorative Materials}

To achieve goals of MID, modern concepts propose only minimal removal of demineralized 'infected' dentin, leaving affected dentine to be restored by a material that adheres to tooth surface and remineralize it.GIC is one such material, which as a reservoir for fluoride not only has the potential to remineralize but can also be recharged with ions from topical fluoride treatments or dentifrices ${ }^{27}$.

Bioactive and bio mimetic materials form a surface layer of an apatite-like material in presence of an inorganic phosphate solution with continuous release of fluoride, thus remineralise tooth.

\section{Future Perspectives}

As the scientific techniques evolve and knowledge progresses, it brings about all new inquisitiveness and pose as many questions as it does answer!

The concept of minimal invasive dentistry has opened arenas of research in several areas. Though demineralization/ remineralization cycle is reasonably clearly defined by Fejerskov; however, methods of reversing cycle and bringing remineralization are yet not clearly understood. ${ }^{78}$ The interrelationship between vast array of micro-organisms present in oral environment needs to research. New techniques are under investigation and there is a need for refinement in understanding of white spot lesion occurrence and its reversal ${ }^{28}$.

There must be thorough investigations into role of saliva in oral disease and ways to improve saliva flow or methods of countering a reduction in flow. This subject has been neglected but has now become relevant particularly in presence of drugs, alone or in combination, which are antisialagogues and pose serious problems, for youth and medically compromised patient ${ }^{29}$.

In recent years, euphoria of aesthetic direct restorations has somehow eclipsed concerns for longevity. There is a definite need for more clinical trials and research; particularly in vivo investigations if problems of longevity of direct restorative materials are to be overcome. Further research is mandated to eliminate setting shrinkage and at same time improvise depth of cure of resins. Glass-ionomer requires continuing research on its ability to enhance remineralization of demineralized tooth structure. The whole concept of ion exchange between tooth structure and cement needs to be investigated and, if possible, improved. The ion exchange adhesion to both enamel and dentine is best method of adhesion available Therefore, these properties need to be enhanced, particularly increased resistance to fracture $^{30}$.

There is a need to address that how much demineralized tooth structure should be removed or saved reliably. Several modifications to the cavity designs suggested by Black have been clinically tested but so far there is no consensus on what is acceptable ${ }^{31}$.

\section{Conclusion}

As predicted by Dr. Black in 1896, the day has arrived when we can practice "Preventive rather 
than Reparative dentistry". "Change" will be key word in dentistry during next few years.

Given that recurrent caries is most common reason for failure of restorations of any type, it is important to move clinical focus away from art and science of restoring teeth ravaged by dental caries to prevention of disease and its early interception. It is not possible to really imitate natural tooth structure on a long-term basis, so it is best that it will be retained as far as possible. While term CAMBRA (Caries Management by Risk Assessment) summarizes one approach, current conceptualization of MID concept now incorporates both maximum intervention as well as minimally invasive treatments. However, use of these technologies will require extensive retraining of clinical dentists; as it will alter way dentists diagnose, intervene, treat, and manage oral diseases. Meanwhile there is an equal need to inform public about benefits of these modern methods of diagnosis, prevention, remineralization, minimal intervention, and repair as compared to conventional restorative procedures as patient's long-term biological and fiscal cost savings will be worth this knowledge ${ }^{32}$.

\section{References}

1. Brostek AM, Walsh LJ. Minimal Intervention Dentistry in General Practice. J of OHDM 2014; Vol 13 (2).

2. Frencken Jo E, Peters MC, Manton DJ, Leal SC, Gordan VL, Eden E. Minimal Intervention Dentistry (MID) for managing dental caries- a review: Report of FDI task group. Int Dent J 2012; 62(5):223-243.

3. Smith DC. J of Biomaterials 1998; Vol 19; Issue 6:467-478.

4. Featherstone, John D.B. Caries prevention and reversal based on the caries balance. Pediatric Dentistry 2006; Vol 28; Issue 2:128-132.

5. Fejeskov O. Changing Paradigms in Concepts of Dental Caries: Consequences for Oral Health Care. Caries Res 2004; 38: 182-191.

6. Featherstone JD. The caries balance: the basis for caries management by risk assessment. Oral Health \& Preventive Dentistry 2004; Vol 2; Suppl 1:259-264.
7. Dawson AS, Makinson OF. Dental treatment and dental health. Part 1. A review of studies in support of a philosophy of Minimum Intervention Dentistry. Aust Dent J. 1992 Apr; 37(2):126-32.

8. Amaechi BT. Emerging technologies for diagnosis of dental caries: The road so far. J. Appl. Phys. 2009; Vol 10; Issue 10.

9. Bennett T. Arnaechi BT, Loveren CV. Fluorides and Non-Fluoride Remineralization Systems. Monogr Oral Sci. Basel, karger. 2013; Vol 23: 15-26.

10. Clementino-Luedemann TN, Dabanoglu A, Ilie N, Hickel R, Kunzelmann KH. Microcomputed tomographic evaluation of a new enzyme solution for caries removal in deciduous teeth. Dent Mater J. 2006 Dec; 25(4):675-83.

11. Nowak AJ. Using Anticipatory Guidance to Provide Early Dental Intervention.

Journal Of The American Dental Association (1939) 126(8):1156-63.

12. Tinanoff N. Diet and dental caries in preschool children. Dent Clin N Am 2005; 49: 25- 737.

13. Cidro J. Breast feeding practices as cultural interventions for early childhood caries in Cree communities. BMC Oral Health. April 2015; 15 (1).

14. Reynolds EC, Cain CJ, Webber FL, Black CL, Riley PF, Jhonson IH, Perich JW. Anticariogenicity of calcium phosphate complexes of tryptic casein phosphopeptitides in the rat. J Dent Res. 1995; 74(6): 1272-9.

15. Reynolds EC. Remineralization of enamel subsurface lesions by casein phosphopeptide stabilized calcium phosphate solutions. J Dent Res. 1997; 76(9): 1587-95.

16. Reynolds EC. Calcium phosphate-based remineralization systems: scientific evidence? Australian Dental Journal. 2008; Vol 2 (3): Pages 268-273.

17. Schemehorn BR, Wood GD, Winston AE. Laboratory enamel solubility reduction and fluoride uptake from enamelon dentifrice. J Dent Res. 1999; 10(1 spec No): 9-12.

18. Saini R. Ozone therapy in dentistry: A strategic review. Journal of Natural Science, Biology and Medicine. July 2011; Vol 2: Issue 2. 
19. Gordan VV, Riley JL, Geraldeli S, Rindal DB, Qvist V, Fellows JL, Kellum HP, and The DPBRN Collaborative Group. Repair or replacement of defective restorations by dentists in The Dental PBRN. J Am Dent Assoc. 2012 Jun; 143(6): 593-601.

20. Smales RJ, Yip HK. The atraumatic restorative treatment (ART) approach for primary teeth: review of literature. Pediatric Dentistry, 2000, v. 22 n. 4, p. 294-298.

21. Mc Comb D. Conservative Operative Management Strategies. Dent Clin N Am. 2005; (49): 847-865.

22. Summitt JB. Conservative cavity preparations. Dent Clin N Am. 2002; (46): 171-184.

23. Ganesh M, Parikh D. Chemomechanical caries removal agents: Review and Clinical application in primary teeth. J Dent Oral Hyg. 2011; Vol 3: (3), Pge 34-45.

24. Hegde VS, Khatavkar RA. A new dimension to conservative dentistry: Air abrasion. J Conserv Dent. 2010; Vol 13: Issue 1

25. Bader C, Krejc I. Indications, and limitations of Er: YAG laser applications in dentistry. Am J Dent 2006;19: 178-186.
26. Kotlow LA. Lasers in Pediatric Dentistry. Dent Clin N Am. 2004; 48: 889-922.

27. Tyas MJ, Burrow MF. Adhesive restorative materials: A review. Australian Dental Journal 2004; 49: (3):112-121.

28. Murdoch-kinch MA. Minimally invasive dentistry. JADA. 2003; Vol. 134.

29. S Bhasin, P Sudha, T Anegundi R. Chair side simple caries activity test: Ora test. J Indian Soc Pedod Prev Dent. 2006; 24: 76-9.

30. Fross H, Hakme E. Retention of a glass ionomer cement and a resin-based fissure sealant and effect on carious outcome after 7 years. Community dentistry and Oral Epidemiology. 1998; Volume 26: Issue 1, Pages 21-25.

31. Nordbo H, Brown G, Tjan AH. Chemical treatment of cavity walls following manual excavation of carious dentin. Am J Dent. 1996 Apr; 9 (2):67-71.

32. Tyas MJ, Anusavice KJ, Frencken JE, Mount GJ. Minimal intervention dentistry--a review. FDI Commission Project 1-97. Int Dent J. 2000 Feb; 50(1):1-12. 